\title{
Model interpretation of the ionospheric F-region electron density structures observed by ground-based satellite tomography at sub-auroral and auroral latitudes in Russia in January-May 1999
}

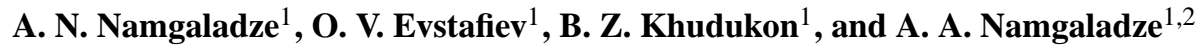 \\ ${ }^{1}$ Polar Geophysical Institute, 15 Khalturina St., 183010 Murmansk, Russia \\ ${ }^{2}$ Murmansk State Technical University, 13 Sportivnaya St., 183010 Murmansk, Russia
}

Received: 8 February 2002 - Revised: 26 September 2002 - Accepted: 18 November 2002

\begin{abstract}
A satellite tomographic campaign was carried out in Russia during January-May 1999. The receiver chain consisted of four sites extending from the north of Karelia to the north of the Kola Peninsula. The F-region electron density measurements were performed during the main seasons (the winter, equinox and summer), and the data contained typical levels of solar activity ( $F_{10.7}$ varied from 100 to 200 ). The magnetic activity was quite low $\left(K_{p}=2-3\right)$. The Upper Atmosphere Model (UAM), the theoretical model of the Earth's atmosphere, as well as two known empirical ionospheric models, IRI-95 and RIM-88, have been applied to compare with experimental data. The tomographic images were interpreted by using simulation results obtained by the models which were also compared to one another. The analysis shows the following: (a) all three models show the best agreement with the tomography data at the height $300 \mathrm{~km}$ (near $h m \mathrm{~F} 2$ ) in comparison with the heights below and above $h m \mathrm{~F} 2(200$ and $400 \mathrm{~km}$ ); (b) all three models systematically underestimate the electron density values in comparison with the tomography data at the height $200 \mathrm{~km}$ and overestimate them at the height $400 \mathrm{~km}$; (c) for all investigated events the $N_{e}(\mathrm{UAM})$ values are closest to $N_{e}$ (tomo) in 399 of 1125 examined data points (36\%), $N_{e}(\mathrm{RIM}-88)$ values are closest to $N_{e}$ (tomo) in 510 cases $(45 \%)$ and $N_{e}$ (IRI-95) values are closest to $N_{e}$ (tomo) in 216 cases (19\%). For the only daytime events, the $N_{e}$ (UAM) values are closest to $N_{e}$ (tomo) in 274 of 624 data points (44\%), whereas $N_{e}$ (RIM-88) daytime values are closest to $N_{e}$ (tomo) in 221 cases (36\%) and closest to $N_{e}$ (IRI-95) values in 129 cases $(20 \%)$. It means that for all events RIM- 88 has the best agreement with the tomography measured electron densities, whereas UAM has the best agreement with the daytime tomography measured electron densities, and IRI-95 has the worst agreement for both daytime and all events; (d) simulated UAM daytime values of electron density near the F2-layer maximum agree with corresponding tomography images for all seasons for
\end{abstract}

Correspondence to: A. N. Namgaladze

(namgaladze@mstu.edu.ru) the first half of 1999, covering almost the total range of the solar activity, so that no correction of the solar EUV flux (used as an input parameter in the UAM) is required; (e) a necessary correction of simulated precipitating soft electron flux intensities has to be made, in order to improve the consistency between measured night-time values of the electron density and those estimated by the theoretical model; (f) the simulated electron density behaviour caused by spatial, diurnal, seasonal variations, as well as due to a solar activity is consistent with the experimental tomographic images. This indicates a good reliability of both experimental and simulated data (at least in the central part of the examined latitudinal interval).

Key words. Ionosphere (auroral ionosphere; modeling and forecasting)

\section{Introduction}

The satellite ionospheric radio tomography and the 3-Dupper atmosphere modelling are the most modern tools in studies of the Earth's ionosphere. They are rather new and represent rapidly developing methods in geophysical studies. It is of great interest and importance to compare experimental electron density data with the results obtained by both theoretical and empirical (statistical) models of the ionosphere. Initially, such a comparison was made by Namgaladze et al. (2000b) for five satellite tomography images of ionospheric electron density observed over Scandinavia during the Russian-Finish campaign in November 1995 (Nygren et al., 1995). A good analogy has been achieved by comparing the results obtained by ionospheric tomography, empirical and theoretical models (after correcting the total intensity of the solar EUV flux). However, neither seasonal nor solar activity variations of electron density have been considered in these studies.

An analysis of 38 tomographic images of the electron density in the sub-auroral and high-latitude ionospheric F- 


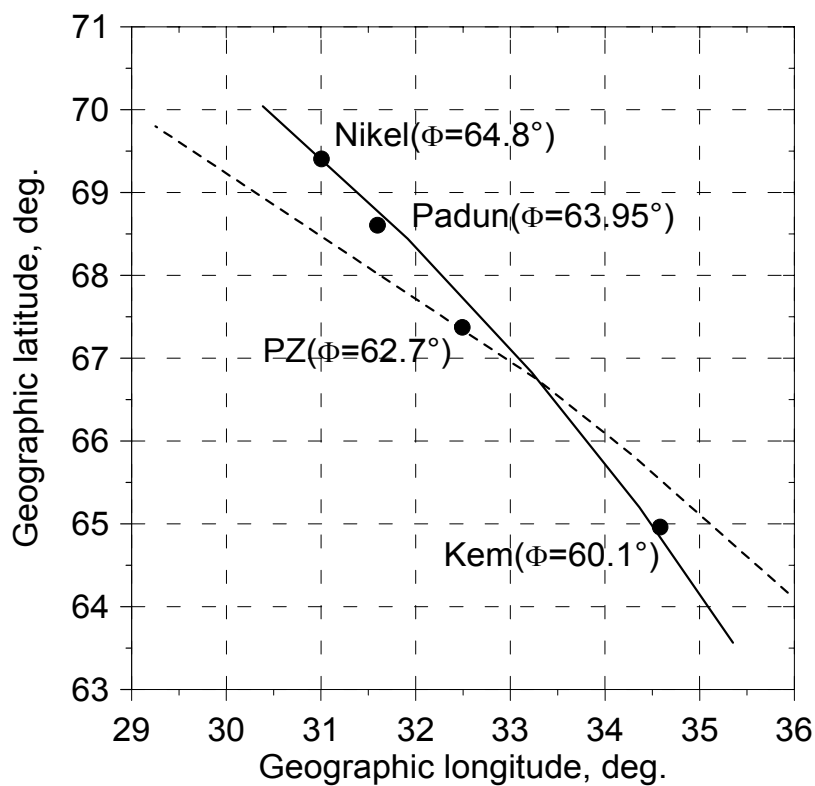

Fig. 1. Tomographic receiver sites (dots) located along the orbit projection of a satellite moving from north (the solid line) and the geomagnetic meridian $\Lambda=125^{\circ}$ (the dashed line). There are the geomagnetic latitudes in the brackets.

regions obtained from satellite ground-based measurements during almost a half-year period from January to May 1999 was performed in the present paper. Simulated results obtained by the global theoretical Upper Atmosphere Model (UAM) (Namgaladze et al., 1998a, b) and two empirical (statistical) models of the ionosphere IRI-95 (Bilitza, 1995) and RIM-88, the Reference Ionosphere Model (Chusovitin et al., 1987), were compared. The observed period covers practically all the seasons and levels of solar activity during rather low magnetic activity. The purpose of the analysis was the following: (1) revealing a consistency between the experimental (tomographic) data and results derived by the theoretical and empirical models with respect to modern physical knowledge about spatial, diurnal, seasonal variations and due to solar activity variations of the electron density in the ionospheric F-region during low geomagnetic activity; (2) defining the UAM's input parameters and estimating their accuracy.

\section{Experimental setup and data}

The tomographic receiver sites located along the orbit projection of a satellite moving from north are presented in Fig. 1. The chain covers the region between $65^{\circ}-69.4^{\circ} \mathrm{N}$, $31^{\circ}-34.6^{\circ} \mathrm{E}$. The figure also shows the geomagnetic meridian plane of $\Lambda=125^{\circ}$ which is used to show the model data. Since the orbit inclination of the satellites is about $83^{\circ}$, that makes the trajectory of satellite movement for a southward passage to lie close to the geomagnetic meridian. The data were converted to F-region electron density maps using a stochastic inversion described by Nygren et al. (1995) and Khudukon (1998). Figure 2 illustrates the changes in the solar and geomagnetic activities (indices $F_{10.7}$ and $K_{p}$ ) during the observations. It can be seen that measurements cover the winter, equinox and summer seasons. The levels of the solar activity varied from $F_{10.7}=100$ to 200 , and the geomagnetic activity was rather low (typically $K_{p}=2-3$ ). This makes it possible to study the spatial, diurnal, seasonal variations of the electron density in the ionospheric F-region, as well as the electron density variations with respect to a solar activity. The following days have been selected for the analysis: 11 January $\left(F_{10.7}=108\right), 18$ January $\left(F_{10.7}=165\right), 1$ February $\left(F_{10.7}=114\right), 14$ February $\left(F_{10.7}=200\right), 22$ March (equinox, $F_{10.7}=115$ ) and 11 May $\left(F_{10.7}=163\right)$. Thus, all seasons and solar activity levels have been presented in the data selected for the analysis.

\section{Model calculations}

Spatial distributions of the electron density for the selected days were derived in the region over the tomographic chain by using the theoretical model UAM (Namgaladze et al., 1998a, b) and two empirical models: the RIM-88 (Chusovitin et al., 1987) and the IRI-95 (Bilitza, 1995). The UAM model is based on the Global Self-consistent Model of Thermosphere, Ionosphere and Protonosphere designed at the Kaliningrad Observatory of IZMIRAN (now the West Department of IZMIRAN) more than 10 years ago (Namgaladze et al., 1988, 1991). The model was further developed and modified at the Polar Geophysical Institute in Murmansk and at the Murmansk State Technical University to be used for the polar upper atmosphere studies (Namgaladze et al., 1998a, b). It describes the Earth's mesosphere, thermosphere, ionosphere, plasmasphere, and the inner part of the magnetosphere confined by the closed geomagnetic field lines as a single system having its electrodynamics.

The model consists of four main computational packages: (1) the package of the neutral atmosphere and lower ionosphere which computes the neutral atmospheric temperature, mass density, neutral gas composition and winds, as well as the ion and electron temperatures, molecular ion density and velocity at altitudes between 60 and $520 \mathrm{~km}$; (2) the package of the ionospheric F2-region and protonosphere computing the ion densities of $\mathrm{O}^{+}$and $\mathrm{H}^{+}$, velocities and temperatures, as well as the electron temperature at altitudes from $175 \mathrm{~km}$ to $15 R_{E}$ of geocentric distance; (3) the electric field package used in calculating the electric field potential both in magnetospheric and thermospheric (dynamo) origins assuming that the geomagnetic field lines are equipotential at altitudes above $175 \mathrm{~km}$; (4) the magnetospheric package which generates magnetospheric plasma-sheet ion density, velocity, pressure and field-aligned currents at the same altitudes used in the second package.

The empirical model of the thermosphere MSISE-90 (Hedin, 1991) was incorporated into the UAM to be used as initial and lower boundary conditions and for calculat- 


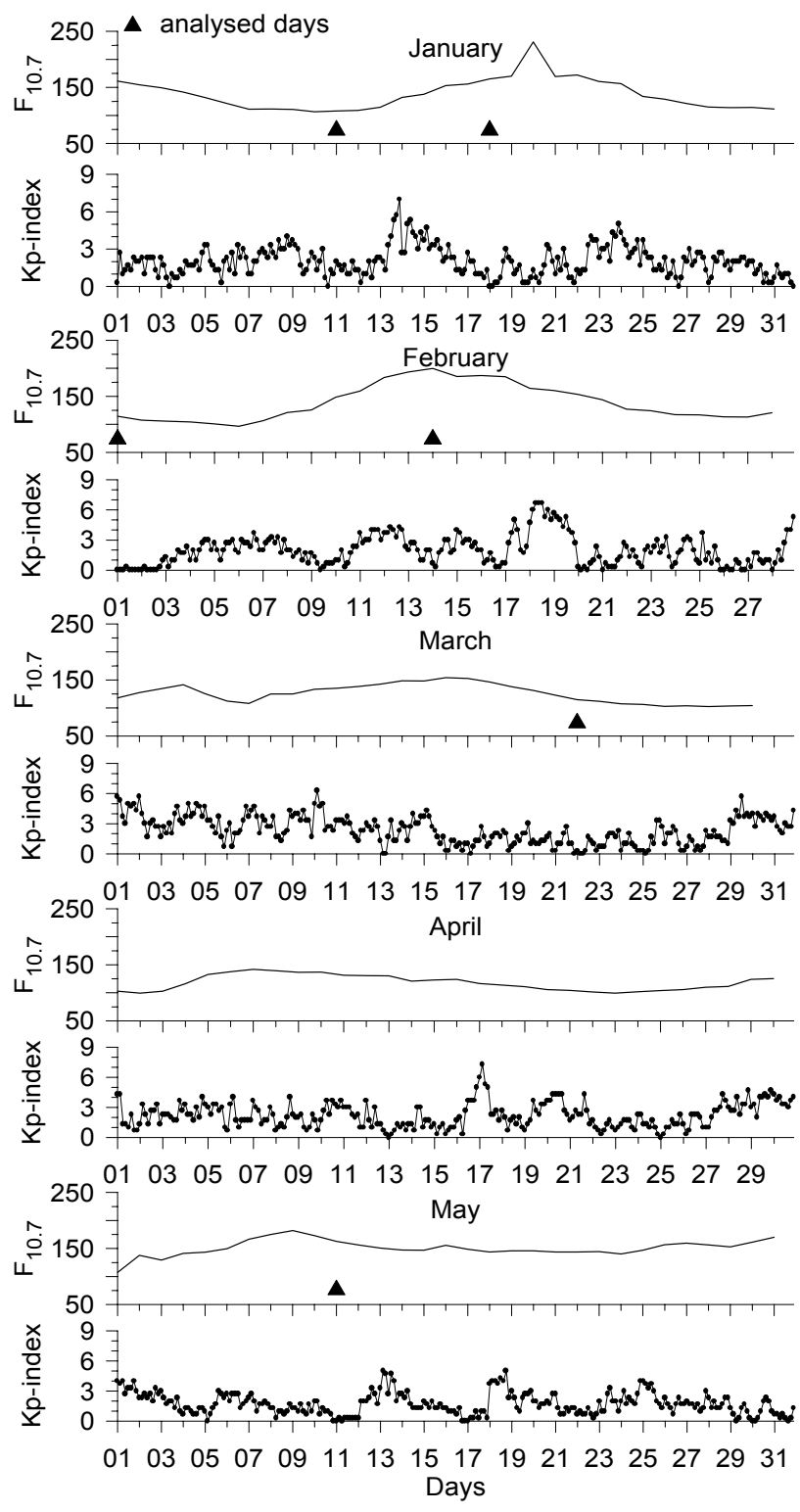

Fig. 2. Variations of the solar and geomagnetic activity during January-May 1999.

ing the neutral gas temperature, density and composition in a global sense at any height in parallel with (or without) self-consistent calculations of these parameters. It makes it possible to compare the results obtained by the theoretical and empirical models of the neutral atmosphere. For the quiet conditions they differ insignificantly at least after one day of numerical integration, whereas they can differ greatly during geomagnetically disturbed conditions (Namgaladze et al., 2000a). In the present study, referring to a low geomagnetic activity, we used the neutral gas temperature, density and composition in MSISE-90 to calculate the variations of the ionospheric parameters.

A version of the UAM was applied, assuming that the input parameters are the electric field potential across the polar cap, having the constant difference of $20 \mathrm{kV}$, and zero field- aligned currents in the zone 2 , corresponding to a low geomagnetic activity. The precipitating cold (average energy $<1 \mathrm{keV})$ and hot $(>1 \mathrm{keV})$ electrons were defined following Hardy's model requirements (Hardy et al., 1985). The maximum flux intensity of the precipitating cold electrons (with the average electron energy of $E_{0}=0.27 \mathrm{keV}$ ) in the region of the daytime cusp was $I_{m}=1.9 \cdot 10^{9} \mathrm{~cm}^{-2} \mathrm{~s}^{-1}$. The maximum intensity $I_{m}$ of hot electrons $\left(E_{0}=3.2 \mathrm{keV}\right)$ is $5 \cdot 10^{8} \mathrm{~cm}^{-2} \mathrm{~s}^{-1}$ at the night-side of the auroral oval and its value for cold electrons $\left(E_{0}=0.5 \mathrm{keV}\right)$ is $4 \cdot 10^{8} \mathrm{~cm}^{-2} \mathrm{~s}^{-1}$. The computational spatial grid has a variable size of the mesh elements along the geomagnetic latitude (from $2^{\circ}$ at about $70^{\circ}$ latitude, to $5^{\circ}$ and $10^{\circ}$ at the equator for obtaining ionospheric and thermospheric parameters, accordingly), a similar size of $15^{\circ}$ in the geomagnetic longitude, and a variable height size (from $3 \mathrm{~km}$ at the $80-\mathrm{km}$ altitude to about $20 \mathrm{~km}$ at the altitude of the F2-layer maximum).

\section{Comparison of the experimental and model data}

Inversion results of the observed electron density in the vertical plane with respect to the geographic latitude are plotted in Figs. 3-8 (the left column of the panels). They have been obtained from the selected tomographic measurements at various times UT. The corresponding simulated electron density plots are presented in the following column order (24 columns from left to right): UAM, RIM-88 and IRI-95. The daytime altitudinal profiles of the electron density at the centre of the examined latitudinal interval $\left(67^{\circ} \mathrm{N}, 33^{\circ} \mathrm{E}\right)$ are shown in Fig. 9. Figure 10 presents the diurnal variations of the observed and simulated values of $N m \mathrm{~F} 2$ and $h m \mathrm{~F} 2$ at the same point in all the selected days.

4.1 Winter electron density during a low solar activity (11 January and 1 February) and a moderate solar activity (18 January)

The electron density plots for these days are shown in Figs. 3-5 where the following features can be seen. Unlike two empirical models, the morning and daytime values of the $N m \mathrm{~F} 2$ peak, the F2-layer thickness, as well as the height distribution (see also Figs. 9 and 10) obtained by the UAM is in a better agreement with the reconstructed images; it can be better seen at 08:53 UT on 11 January, at 11:41 UT on 18 January and at 07:07 UT on 1 February. During low solar activity, the RIM-88 model underestimates $N_{e}$ values on 11 January and 1 February, as compared to the tomographic data and on the contrary, its values are overestimated on 18 January. The IRI-95 electron density values (especially during low solar activity) are overestimated in all the cases and in poor agreement either with the tomographic images or with the other two models. The situation is different near midnight: the electron density values estimated by the IRI-95 are too high compared to the tomographic data. The simulated results obtained by the UAM and the RIM- 88 are both consistent between and with the reconstructed data. The 

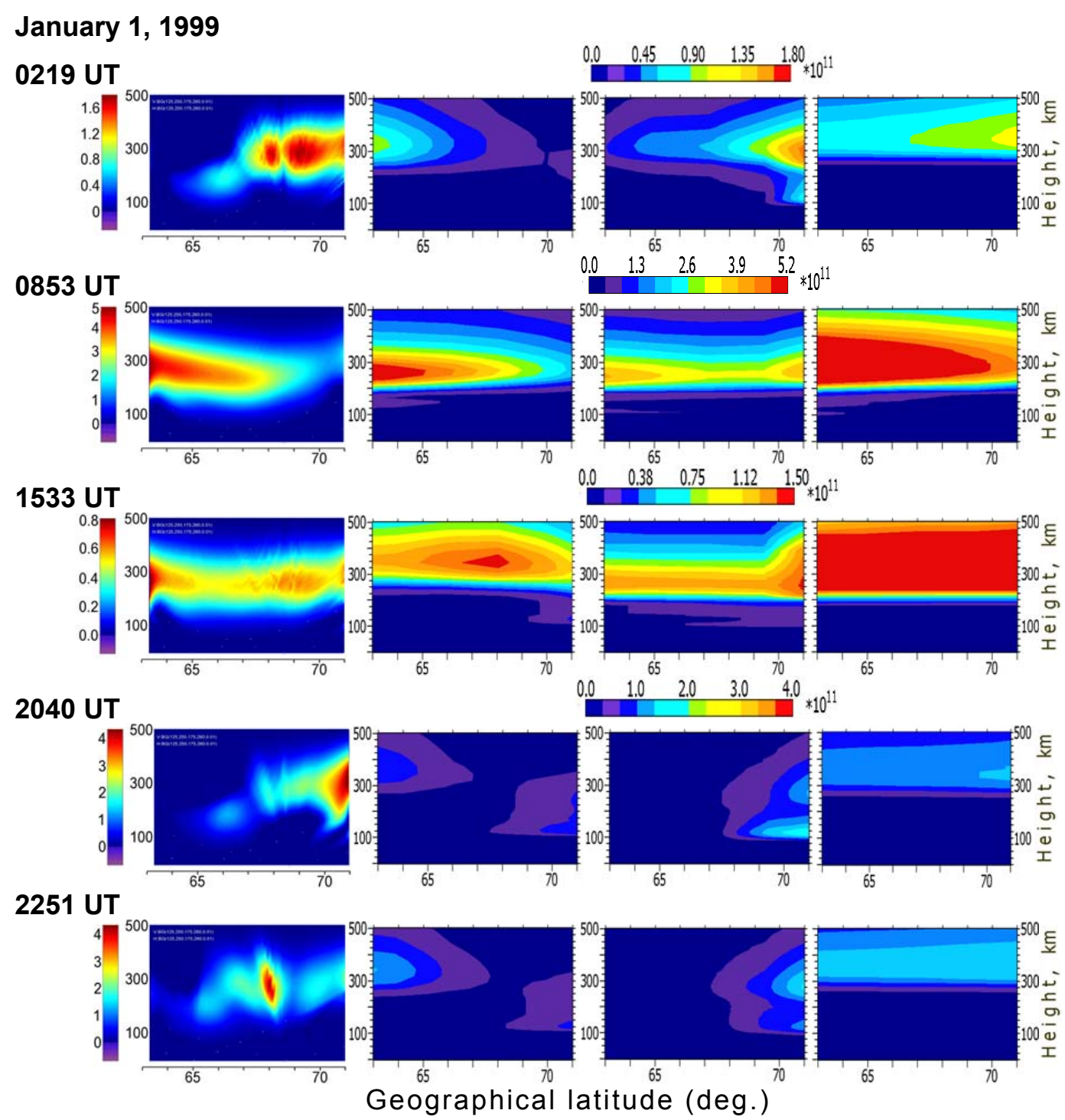

Fig. 3. Tomography images of the electron density $\left(\mathrm{m}^{-3}\right)$ in the vertical plane over the receiver chain (left column) obtained for the observational events on 11 January 1999 (in UT) and the corresponding electron density distributions derived from the models (next three columns from left to right represent the theoretical model UAM, empirical RIM-88 and IRI-95 models, accordingly). All the model calculations were performed in the plane along the geomagnetic meridian of $\Lambda=125^{\circ}$.

above three data sets clearly indicate the trough occurrence, as well as a region with precipitating electrons that can be seen nearby. However, the electron density values in the precipitation region obtained by the UAM is lower compared to the observed ones. This indicates that the intensity of the precipitating cold electrons flux in quiet conditions has to be increased about by about a factor 2 . The essential electron density enhancement in the precipitation region observed in the tomographic images after 18:00 UT on 18 January can be related to a growing magnetic activity up to $K_{p}=3$ in the evening sector. A prominent feature of the tomographic plots should be noted: a plasma enhancement is seen between $67^{\circ}$ and $68^{\circ}$ in the evening sector. The enhancement is not obviously related to a particle precipitation. Similar electron density enhancements are also observed in the UAM re- sults (at 15:33 UT on 11 January, at 15:35 UT on 18 January and at 16:41 UT on 1 February). They can be interpreted to be caused by the plasma transport due to a magnetosphereionosphere convection.

\subsection{Winter electron density during a high solar activity (14 February)}

The highest solar activity occurred on 14 February (Fig. 6), when the $F_{10.7}$-value was about 200, i.e. twice exceeding the level of solar activity of the days discussed above. The following features of the tomographic and model images can be revealed in this case.

The electron density peak values of the tomographic data and the results derived by both models the UAM and the 

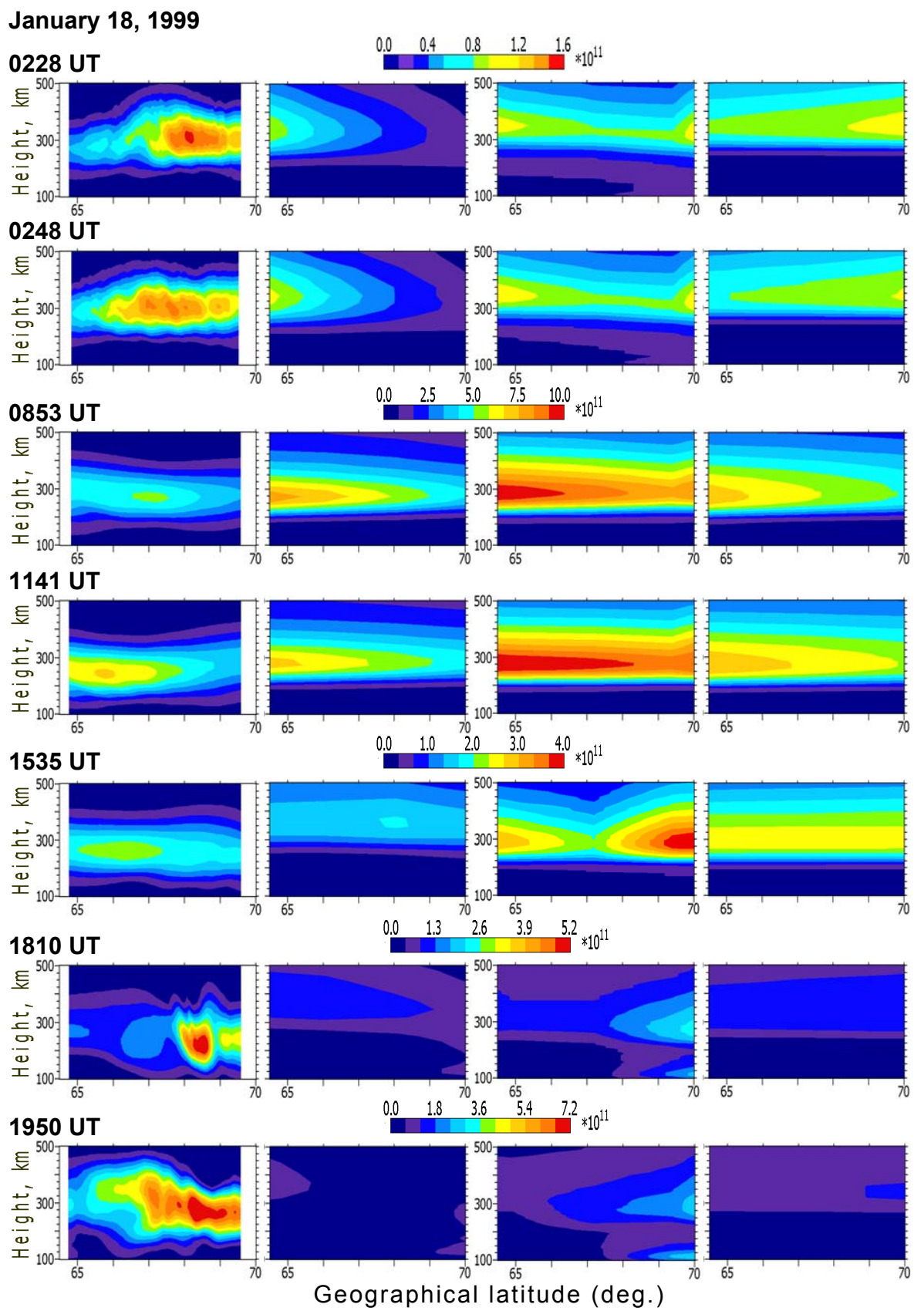

Fig. 4. Same as Fig. 3, on 18 January 1999.

RIM-88, are about twice higher at about 11:43 UT in the daytime sector than in the above cases. Unlike the periods of low solar activity, the IRI-95 model does not contain very high electron density. It can be seen that in the evening and night-side sectors the equatorial boundary of the precipitating electron region was shifted from $69^{\circ}$ to the latitudes of $66^{\circ}-67^{\circ}$ at times of high solar activity with increasing density peak values by about 1.5 in the precipitation region. This requires that intensities of precipitating cold electron fluxes have to be corrected (increased by at least by a factor 3), and the model latitude of the particle precipitation maximum has to moved to the equator by $2-3$ degrees.
4.3 Electron density during a low solar activity on the equinox (22 March)

Figure 7 presents the data obtained for the equinox. In the morning and daytime sectors the tomographic observations and the theoretical model data show a reasonable agreement (see also Figs. 9 and 10); however, both empirical models underestimate the level of the electron density in the daytime. Both tomographic and UAM electron densities have about twice higher values on the equinox compared to the winter-time with similar other conditions. The reconstructed images in the pre-midnight sector (at 19:24 and 20:12 UT) 

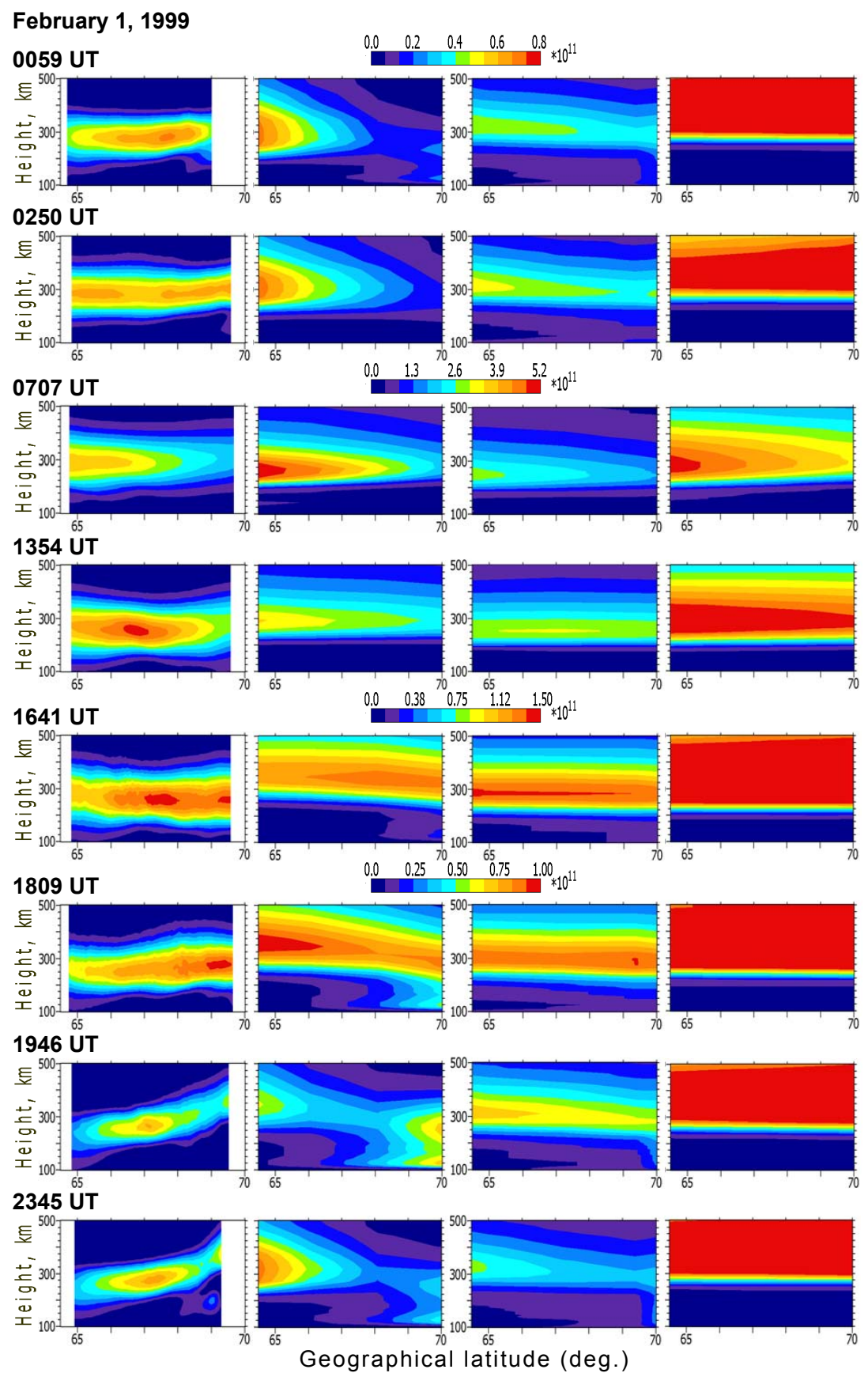

Fig. 5. Same as Fig. 3, on 1 February 1999.

seem to be unusual. They don't agree with any model due to extremely high electron density values on the equatorial wall of the trough, which can be caused by both the convection and precipitation.

\subsection{Electron density during a moderate solar activity in summer (May 11)}

The inversion and model results on 11 May are shown in Fig. 8. As expected, a distinctive feature seen in observations and model plots during summer conditions is the lack of the trough. It seems that the tomographic patterns of this day have inaccuracies at the edges of the examined latitudinal interval due to the shapes in the form of contour lens in these regions. A similar feature was also noticed on some previous observational days. Such shapes are seen neither in the theoretical nor in the empirical ionosphere models and this feature contradicts a property that in the summertime, the electron density is determined mainly by the Sun's zenith angle over all the other physical factors.

In the central part of the studied region the electron density values are slightly overestimated in the UAM patterns com- 


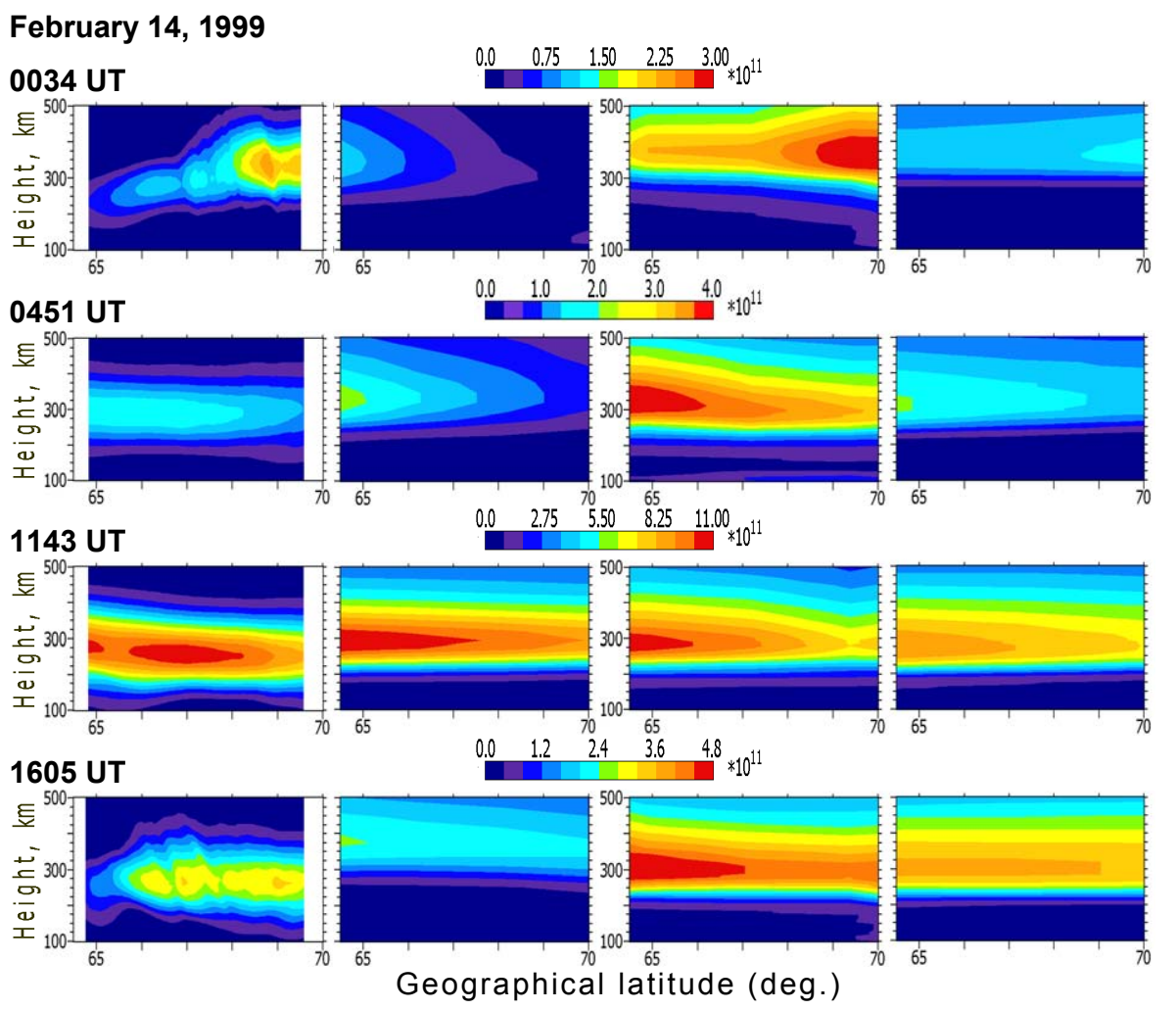

Fig. 6. Same as Fig. 3, on 14 February 1999.

pared to the daytime tomographic images (see also Fig. 9 at 15:22 UT) and vice versa they are underestimated in the morning and evening time. The electron density obtained by both empirical models is underestimated relative to the tomographic values. Relating to the seasonal effect, a comparison of the data obtained during different seasons (11 May, 22 March, 11, 18 January, and 1 February) shows that the seasonal effect on 11 May is composite with the solar activity effect. In particular, the observed and theoretical model values of the electron density in the daytime summer are about twice higher than in the daytime winter and they are almost similar to the values on the equinox. However, the solar activity level in summer is about 1.5 times higher than in the equinox. Therefore, in summer the density values can be possibly predicted to be lower than on the equinox during similar levels of the solar activity. This is in reasonable agreement with known studies referring to seasonal and semi-annual variations of the electron density in the ionospheric F2-region.

4.5 Analysis of the ratios of the model electron density to the electron density measured by the radio tomography

To estimate quantitatively the degree of agreement or disagreement of the model and tomography measured electron densities, we have calculated the ratios of the model electron density to the electron density measured by the radio tomography, $R=N_{e}$ (mod) $/ N_{e}$ (tomo), for 1125 data points. Four electron density values - from three models and tomography data - have been taken to calculate these ratios at every point corresponding to the fixed latitude, altitude and time value at ten latitudes in the range $65.0-69.5^{\circ} \mathrm{N}$ with the step $0.5^{\circ}$ at three heights 200, 300 and $400 \mathrm{~km}$ for 38 tomography images obtained during January-May 1999 period.

The results of these calculations are demonstrated in Fig. 11 which shows histograms of the $N_{e}$ (mod)/ $/ N_{e}$ (tomo) ratios for three models: the theoretical model UAM (left column) and two empirical models RIM-88 (middle) and IRI95 (right column). The histogram bins have been defined for the values of $\lg R$ (a bin size equal to 0.2). Figure 11a shows the ratio distributions for all 1125 data points, whereas Fig. 11b shows them at every height separately (375 data points for every height). Open bars indicate the ratio distributions for all events (1125 data points for all heights or 375 data points for every height), whereas shaded bars correspond only to daytime events (624 data points for all heights or 208 data points for every height). The following features of the ratio distributions are seen clearly from these figures. For all events (open bars in Fig. 11a) the highest bars correspond to the ratio range $R=0.50-0.79(\lg R=$ $-0.3 \div-0.1)$ for UAM and RIM-88 and $R=0.79-1.26$ $(\lg R=-0.1 \div+0.1)$ for IRI-95 whereas for daytime events only (shaded bars in Fig. 11a) the highest bars correspond to $R=0.79-1.26$ for all three models and the height of the highest shaded bars is maximal for UAM and minimal for IRI-95. Analysing all events together we obtain that among three models the $N_{e}$ (UAM) values are closest 
March 22, 1999
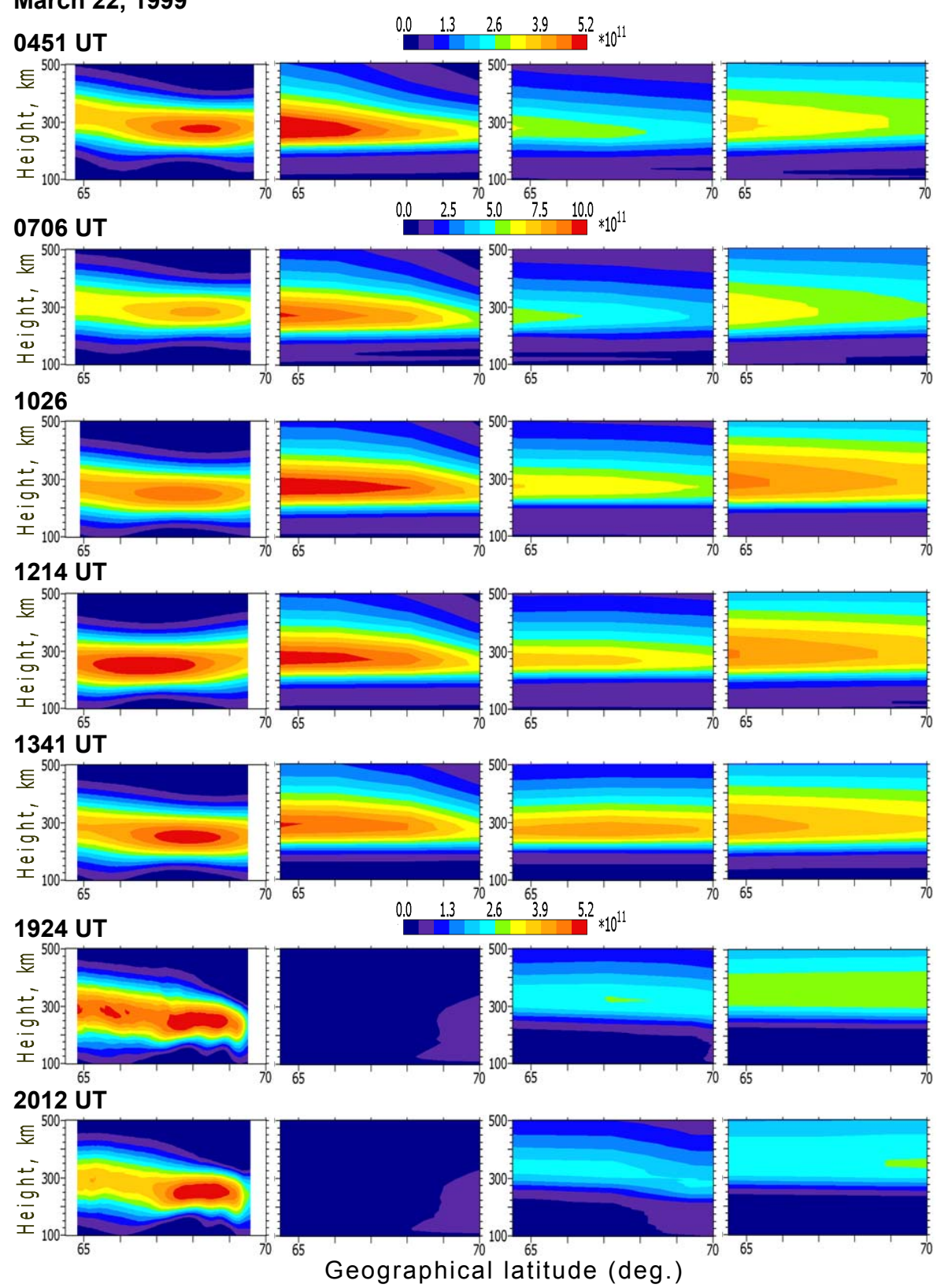

Fig. 7. Same as Fig. 3, on 22 March 1999.

to $N_{e}$ (tomo) in 399 out of 1125 cases $(36 \%), N_{e}$ (RIM88 ) values are closest to $N_{e}$ (tomo) in 510 of 1125 cases (45\%) and $N_{e}$ (IRI-95) values are closest to $N_{e}$ (tomo) in 216 out of 1125 cases (19\%). For the only daytime events, the $N_{e}$ (UAM) values are closest to $N_{e}$ (tomo) in 274 out of 624 cases $(44 \%)$ whereas $N_{e}$ (RIM-88) daytime values are closest to $N_{e}$ (tomo) in 221 cases $(36 \%)$ and closest to $N_{e}$ (IRI-95) values are closest to $N_{e}$ (tomo) in 129 out of 624 cases $(20 \%)$. It means that for all events RIM- 88 has the best agreement with the tomography measured electron densities, whereas UAM has the best agreement with the daytime tomography measured electron densities, and IRI-95 has the worst agreement for both daytime and all events.
At the fixed height $200 \mathrm{~km}$ (the top panel in Fig. 11b), the highest open bars correspond to $R=0.50-0.79$ for UAM (as well as the highest shaded bars), $R=0.32-0.50$ for RIM-88 (as well as the highest shaded bars), and $R=$ $0.20-0.32$ for IRI-95 $(R=0.20-0.50$ for the highest shaded bars). It means that all three models systematically underestimate the $N_{e}$ values in comparison with $N_{e}$ (tomo) at this height for both daytime and all events, and UAM has the minimal disagreement, whereas IRI-95 has the maximal disagreement.

At the fixed height $300 \mathrm{~km}$ (the middle panel in Fig. 11b) the highest open bars correspond to $R=0.79-1.26$ for UAM and IRI-95, and to $R=0.50-0.79$ for RIM-88. The 
May 11, 1999
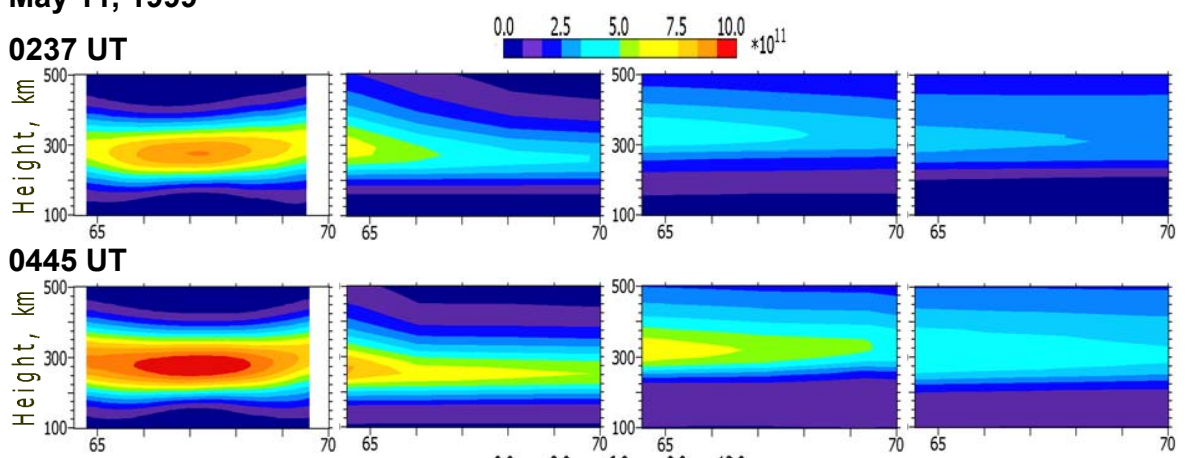

0445 UT

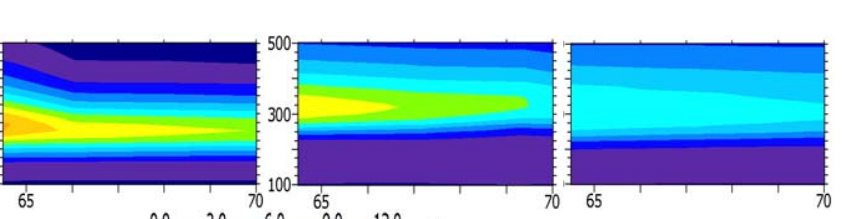

0645 UT
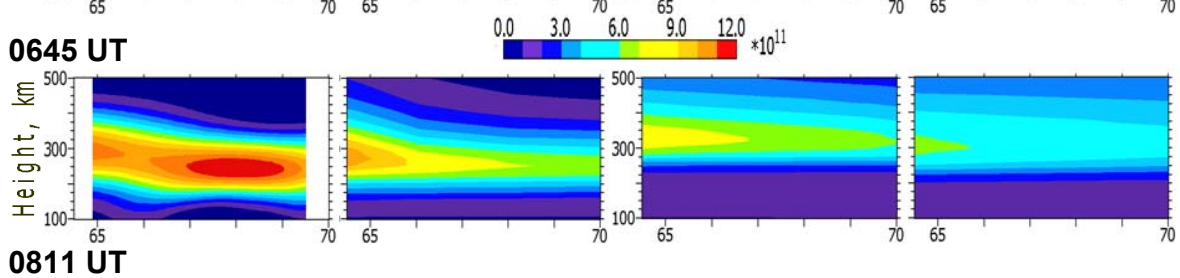

0811 UT
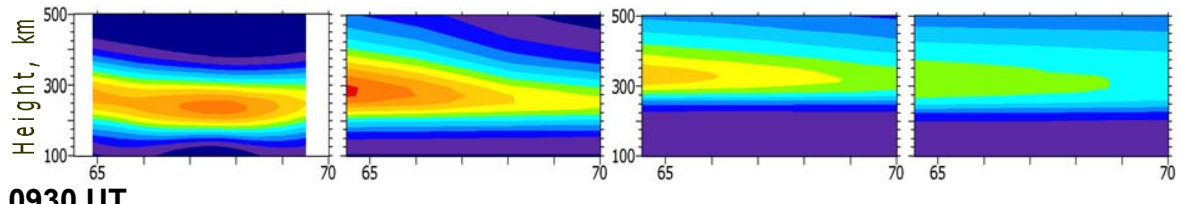

0930 UT
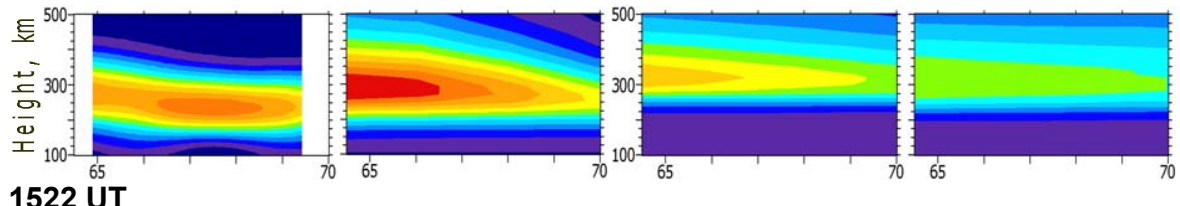

1522 UT
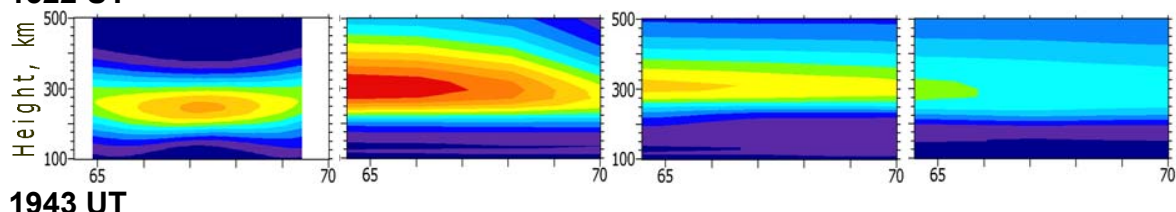

1943 UT
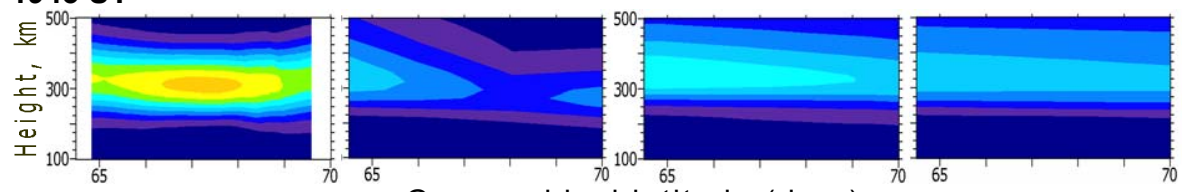

Geographical latitude (deg.)

Fig. 8. Same as Fig. 3, on 11 May 1999.

highest shaded bars correspond to $R=0.79-1.26$ for all three models at this altitude, and the height of the highest shaded bar is maximal for UAM and minimal for IRI-95, but the differences between these shaded bar heights are insignificant. We see in Fig. $11 \mathrm{~b}$ that for the height $300 \mathrm{~km}$ all three models show the best agreement with $N_{e}$ (tomo) in comparison with other heights (200 and $400 \mathrm{~km}$ ).

At the fixed height $400 \mathrm{~km}$ (the bottom panel in Fig. 11b) the highest open bars (as well as the highest shaded bar) correspond to $R=2.0-3.2$ for UAM and IRI-95, and $R=3.2-5.0$ for RIM-88. It means that all three models systematically overestimate the $N_{e}$ values in comparison with $N_{e}$ (tomo) at this height, and UAM has the minimal disagreement, whereas RIM-88 has the maximal disagreement at this height.

As for the $h m \mathrm{~F} 2$ and $N m \mathrm{~F} 2$ values at the centre of the investigated latitudinal interval we can see from Fig. 10 that all three models systematically overestimate $h m \mathrm{~F} 2$, giving values of about $250-350 \mathrm{~km}$ instead of about $250-300 \mathrm{~km}$ given by the tomography, whereas $N m \mathrm{~F} 2$ from UAM are rather close to the tomography values. Summarizing, we can conclude that:

1. All three models show the best (and approximately similar) agreement with $N_{e}$ (tomo) at the height $300 \mathrm{~km}$ (near the F2-layer maximum height) in comparison with the heights below and above $h m \mathrm{~F} 2(200 \mathrm{~km}$ and $400 \mathrm{~km})$; 

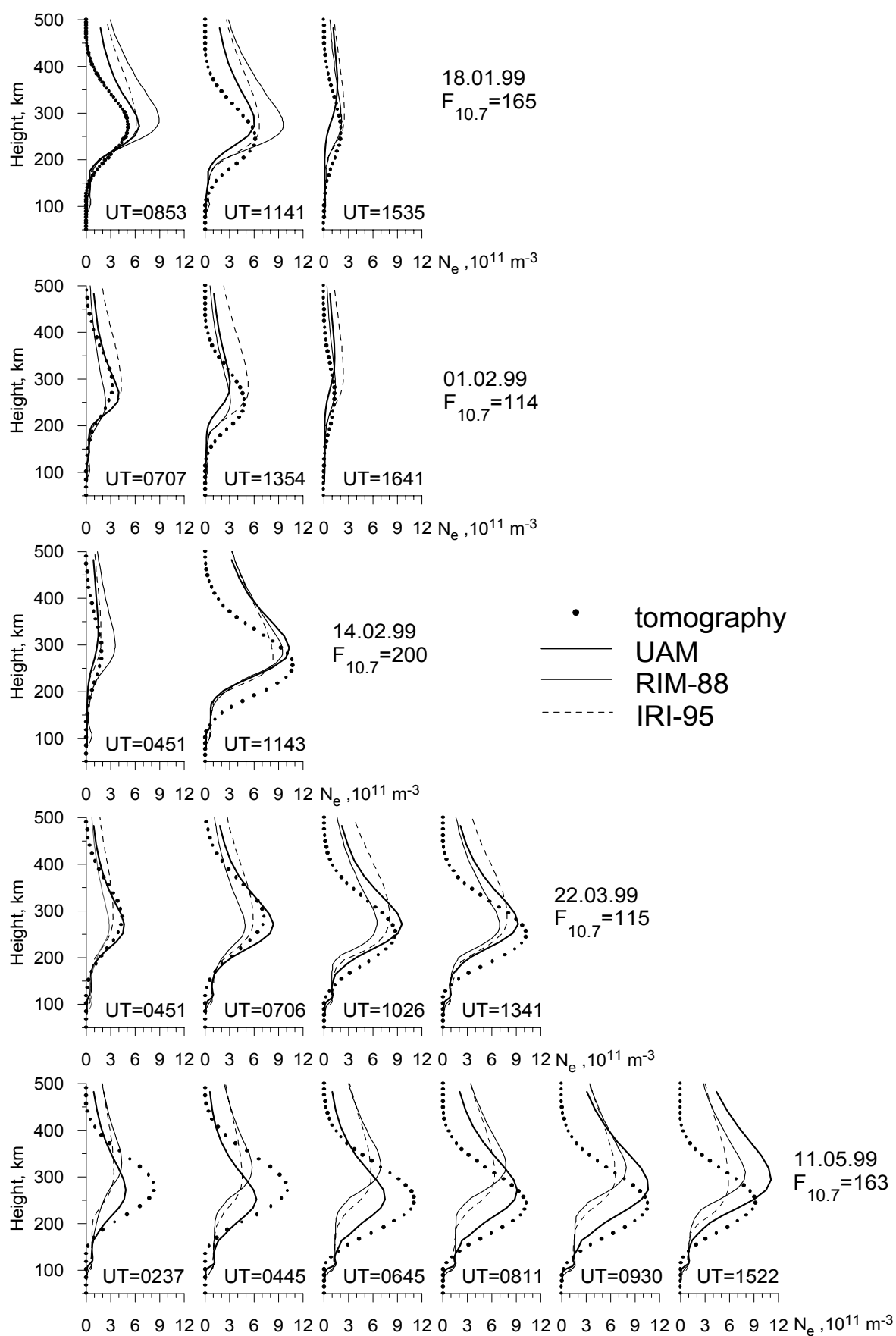

Fig. 9. Height profiles of the electron density $N_{e}\left(\times 10^{11} \mathrm{~m}^{-3}\right)$ extracted from the reconstructed and simulated data in the centre of the examined region $\left(\varphi=67^{\circ} \mathrm{N}\right)$ in the daytime sector of the selected cases.

2. All three models systematically underestimate the $N_{e}$ values in comparison with $N_{e}$ (tomo) at the height $200 \mathrm{~km}$ and overestimate them at the height $400 \mathrm{~km}$;

3. For all events and all heights RIM- 88 has the best agreement with the tomography measured electron densities, whereas UAM has the best agreement with the only daytime tomography measured electron densities, and IRI95 has the worst agreement for both only daytime and all events.

\section{Conclusions}

Selected cases of the electron density changes in the ionospheric F-region over the Kola Peninsula and Karelia in Russia at latitudes from $63^{\circ}$ to $71^{\circ} \mathrm{N}$ observed by satellite radio tomography between January and May 1999 have been interpreted by using the global theoretical model of the upper Earth's atmosphere (UAM), as well as by two empirical models of the ionosphere (IRI-95 and RIM-88). Spatial (height- 


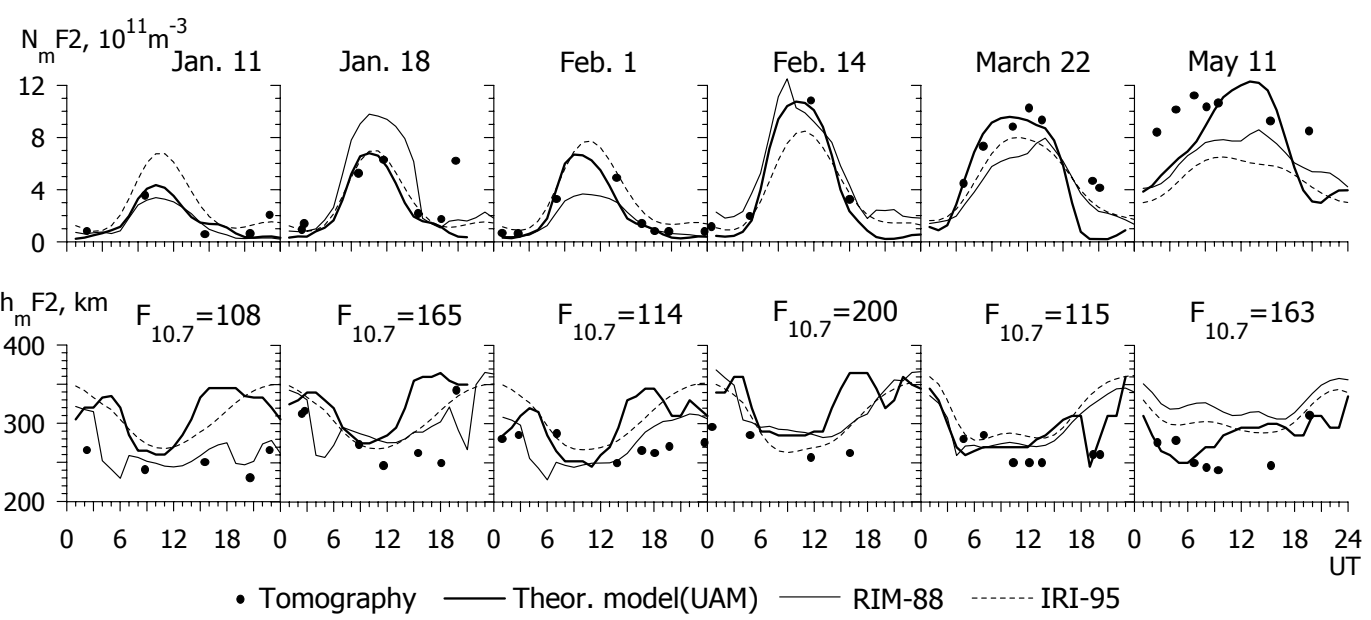

Fig. 10. Diurnal variations of $N m \mathrm{~F} 2$ and $h m \mathrm{~F} 2$ at $67^{\circ} \mathrm{N}, 33^{\circ} \mathrm{E}$ in January-May 1999 from tomography and model calculation data.

(a) for all three heights together

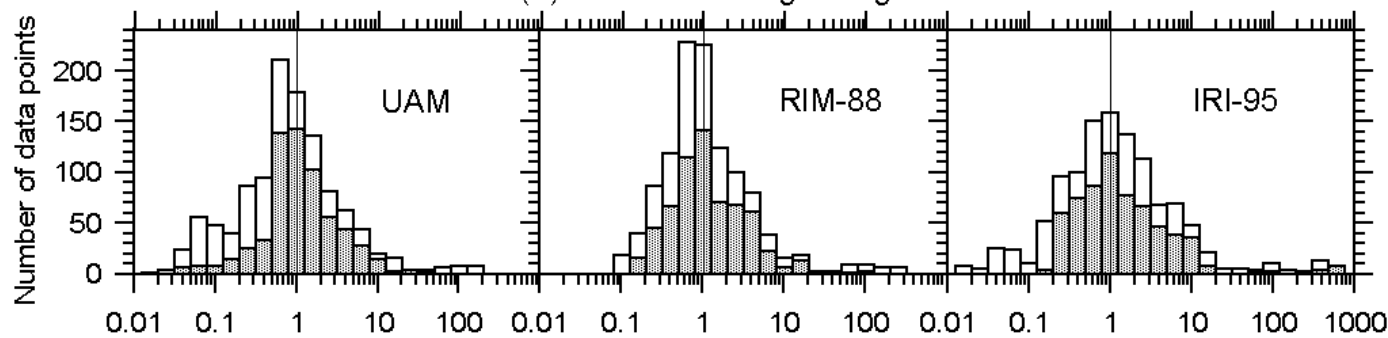

(b) for every height separately

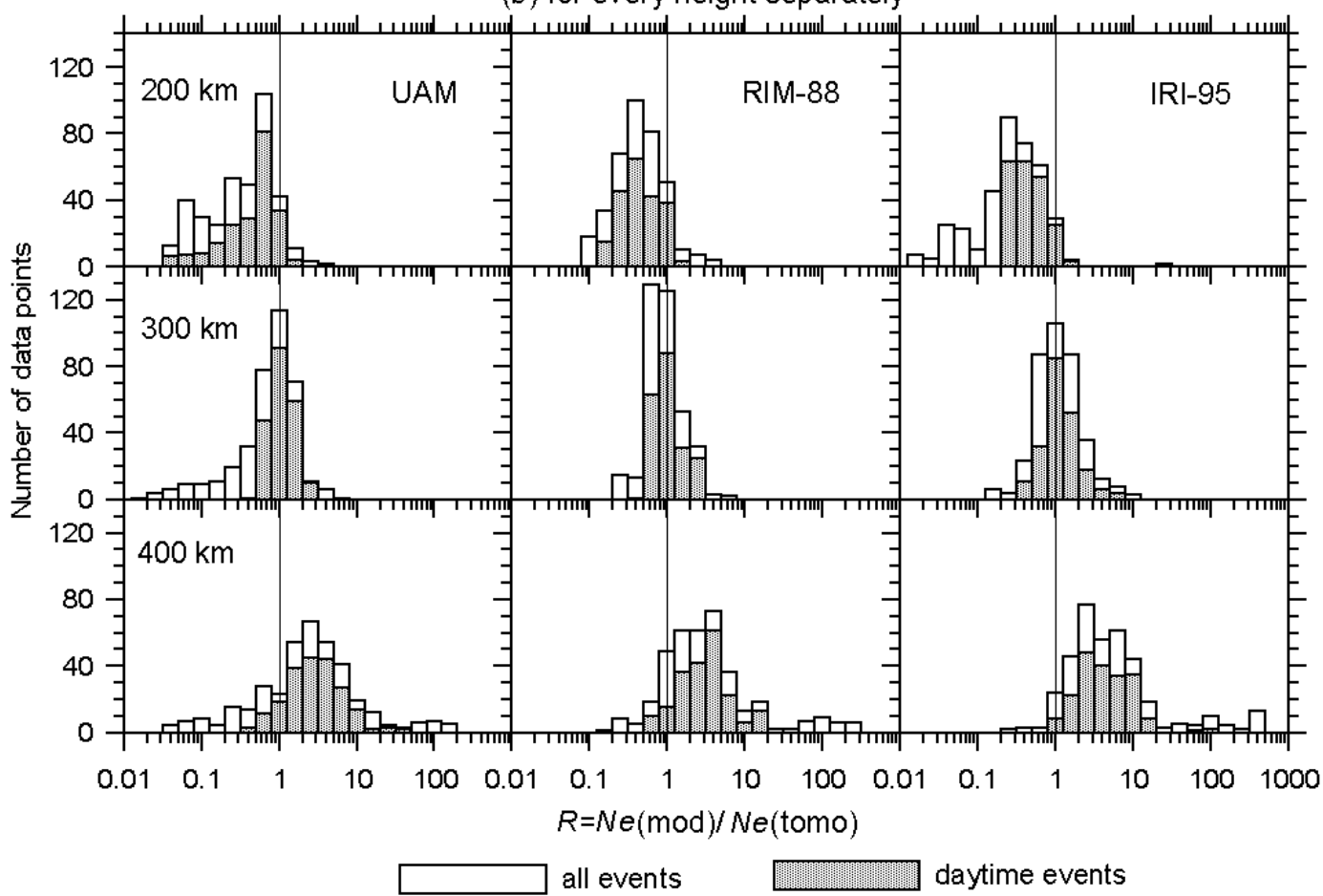

Fig. 11. Histograms of the ratios of the model electron density to the electron density measured by the radio tomography, $R=$ $N_{e}(\bmod ) / N_{e}$ (tomo), for three ionosphere models: the theoretical model UAM (left column) and two empirical models RIM-88 (middle column) and IRI-95 (right column). (a) For all three heights together (200, 300 and $400 \mathrm{~km})$. (b) For every height separately. 
latitudinal), diurnal and seasonal variations of the electron density, as well as its changes due to the solar activity during a low geomagnetic conditions were discussed.

Summarising the results of the comparison between the three models and tomography measured electron density data, we can conclude that all three models show the best agreement with $N_{e}$ (tomo) at the height $300 \mathrm{~km}$ (near $h m \mathrm{~F} 2$ ) in comparison with the heights below and above $h m \mathrm{~F} 2(200$ and $400 \mathrm{~km}$ ). All three models systematically underestimate the $N_{e}$ values in comparison with $N_{e}$ (tomo) at the height $200 \mathrm{~km}$ and overestimate them at the height $400 \mathrm{~km}$. For all events and all heights RIM- 88 has the best agreement with the tomography measured electron densities, whereas UAM has the best agreement with the only daytime tomography measured electron densities, and IRI-95 has the worst agreement for both only daytime and all events. The UAM daytime electron density values near the F2-layer maximum height are quite consistent with the corresponding tomographic images during all the observed seasons (see, e.g. Figs. 9 and 10); therefore, no correction of the solar EUV flux used in the model is required.

The intensity of the precipitating soft electron fluxes used in the model has to be corrected (increased by the factor of 2-3 in quiet conditions), in order to improve the agreement between night-time values of the electron density observed by tomography and simulated by the UAM. The latitudinal dependence of the precipitation maximum on the solar activity must also be included into the model. The main changes of the observed electron density (spatial, diurnal, seasonal and due to a solar activity) can be simulated and interpreted by the UAM, showing its reasonable accuracy, at least in the central part of the latitudinal range under study.

Acknowledgements. This work was supported by the Grants No.0005-65132 and No. 00-05-65511 of the Russian Foundation for Basic Research.

Topical Editor M. Lester thanks a referee for his help in evaluating this paper.

\section{References}

Bilitza, D.: The high latitudes in the international reference ionosphere: summary, Adv. Space Res., 16, 1, 1995.

Chusovitin, Yu. K., Shirochkov, A. V., Besprozvannaya, A. S., Gulyaeva, T. L., Denisenko, P. F., Armenskaya, O. A., Ivanova,
S. E., Kashirin, A. I., Klueva, N. M., Koryakina, E. A., Mironova, L. S., Sykilinda, T. N., Shushkova, V. B., Vodolazkina, V. I., Sotsky, V. V., and Sheidakov, N. E.: An empirical model for the global distributions of density, temperature and effective collision frequency of electrons in the ionosphere, Adv. Space Res., 7, 49, 1987.

Hardy, D. A., Gussenhoven, M. S., and Holeman, E.: A statistical model of auroral electron precipitation, J. Geophys. Res., 90, 4229, 1985.

Hedin, A. E.: Extension of the MSIS thermosphere model into the middle and lower atmosphere, J. Geophys. Res., 96, 1159, 1991.

Khudukon, B. Z.: Bayesian probabilistic approach to ionospheric radiotomography, (Review), in: Modelling processes in the upper polar atmosphere, Murmansk, 63, 1998.

Namgaladze, A. A., Korenkov, Yu. N., Klimenko, V. V., Karpov, I. V., Bessarab, F. S., Surotkin, V. A., Glushchenko, T. A., and Naumova, N. M.: Global model of the thermosphere-ionosphereprotonosphere system, Pure and Applied Geophysics, 127, 219, 1988.

Namgaladze, A. A., Korenkov, Yu. N., Klimenko, V. V., Karpov, I. V., Surotkin, V. A., and Naumova, N. M.: Numerical modelling of the thermosphere-ionosphere-protonosphere system, J. Atmos. Terr. Phys., 53, 1113, 1991.

Namgaladze, A. A., Martynenko, O. V., Volkov, M. A., Namgaladze, A. N., and Yurik, R. Yu.: High-latitude version of the global numerical model of the Earth's upper atmosphere, Proceedings of the MSTU, (http://www.mstu.edu.ru/publish/ vestnik), 1, 2, 23, 1998a.

Namgaladze, A. A., Martynenko, O. V., and Namgaladze, A. N.: Global model of the upper atmosphere with variable latitudinal integration step, Geomagnetism and Aeronomy International, 1, 53, 1998b.

Namgaladze, A. A., Foerster, M., and Yurik, R. Yu.: Analysis of the positive ionospheric response to a moderate geomagnetic storm using a global ionospheric model, Ann. Geophysicae, 18, 461, 2000a.

Namgaladze, A. A., Namgaladze, A. N., and Yurik, R. Yu.: Global modeling of the quiet and disturbed upper atmosphere, Phys. Chem. Earth (C), 25, 533, 2000b.

Nygren, T., Markkanen, M., Lehtinen, M., Pirtilla, J., Henelius, P., Vilenius, E., Tereshchenko, E. D., and Khudukon, B. Z.: Bayesian approach to satellite radio tomography with applications in the Scandinavian sector, Ann. Geophysicae, 13, 1277, 1995.

Nygren, T., Tereshchenko, E. D., Khudukon, B. Z., Evstafiev, O. V., Lehtinen, M., and Markkanen, M.: Mapping the ionospheric F-region by means of satellite tomography, Acta Geod. Geoph. Hung., 32, 395, 1997. 